SOMIMAIRE. - J. LE GMLL : L'Alimentation des jeunes Truites en piscicullure pratique. J. L: Gall : I influence de la lumière sur les alevins de salmonides. De Necrbourg: Carpiculture extensive ou intensive. - Chrosique : La pourriture des branchies chez la Carpe. - Recherches sur les dommages occasionnés aux Poissons par les turbines. - Les dommages causés par l'hiver, en Saxe, à la pêche et à la pisciculture. - Découverte récente, en Norvège, d'un Saumon sédentaire. - Commesicitioxs conponstrves: Fédération des Syndicats d'étangs de la région de l'Est. - Syndicat des étangs de la Marne. - Syndicat les étangs de la Neuse. - Syndicat des étangs de Sologne. - Syndicat des étangs du Forez. - Chambre syndicale des étangs de 'Touraine et d'injou. - Syndicat des pisciculteurs de Franche-Comté et de Bourgogne. - Consiztatioss teciniques. - Revseigxements

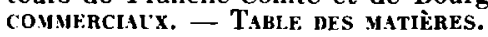

\title{
L'ALIMENTATION DES JEUNES TRUITES EN PISGICULTURE PRATIQUE
}

\author{
Par M. Jean LE GALL \\ Directeur de la Station aquicole de Boulogne-sur-Mer.
}

Le problème est d'importance ; non seulement pour la Truite, mais pour tout poisson d'élevage. Que cherche tout pisciculteur? sinon d'avoir le rendement maximum dans le minimum de temps, autrement dit d'obtenir, dans le plus bref délai possible et avec le minimum de frais, grâce à un régime alimentaire bien compris, la transformation de ses alevins d'élevage en Poissons de taille marchande.

Il est évident que tout pisciculteur de métier a sa méthode qu'il juge la meilleure ou, tout au moins, comme susceptible de lui procurer le meilleur rendement. Aussi ne discuterons-nous pas sur les régimes alimentaires employés par les uns ou les autres. Mais, il apparait de plus en plus qu^, si le même régime ne convient pas à des espèces différentes, et ceci est de toute évidence, les diverses variétés d'une même espèce, la Truite par exemple, ne se comportent pas de la même façon au point de vue rendement : - c'est-à-dire résistance aux maladies et rapidité de croissance, - vis-à- is du même régime. Même pour une scule variété, la valeur relative d'un régime, son " efficience ", pour employer ce néologisme, varie avec l'âge des alevins.

Ces faits ont déjà dû ètre pratiquement constatés par les éleveurs ; ils ont, en outre, pu être suivis et vérifiés expérimentalement aux EtatsUnis, où de nombreuses Stations expérimentales d'élevage, dégagées de tout souci matẻriel d'existence, peuvent suivre d'une façon rigoureusement scientifique l'importance et la valeur relative des différents régimes alimentaires en pisciculture pratique.

Nous ne possédons pas chez nous, de tels établissements. Il est heureusement possible, pour le pisciculteur français, de se tenir au courant des 
résultats de ces recherches longues et minutieuses, grâce à la publication par le Bureau of Fisheries, de Washington, des rapports de ses différentes Stations expérimentales ( 1 ). Nous en avons déjà résumé une partie, dans un précédent travail (2) en montrant l'importance des vitamines dans l'alimentation des alevins d'élevage.

Les travaux poursuivis à la Station expérimentale d'Holden ont particulièrement porté sur la valeur alimentaire des différents régimes pour l'alimentation des alevins des diverses variétés de Truites ; les résultats obtenus ne manquent pas d'intérêt.

Les récrimes habitucllement employés dans les différents établissements de pisciculture des Etats-Unis furent d'abord expérimentés quant à leur valeur relative.

Ces régimes sont normalement constitués par du coeur de bœuf, dı foie de bocuf ou du foie de mouton. Différents essais furent donc tentés avec l'un ou l'autre et il fut prouvé que le "foie de boeuf " constituait le meilleur régime quand l'éleveur envisageait l'élevagae, non pas seulement de l'alevin de repeuplement, mais au delà de six mois, voire jusqu’à la Truite de portion.

A six mois, il fut prouvé, pour la Truite de rivière, qu un régime au foie de bouf produisait une croissance deux fois plus forte qu'un régime au foie de mouton, tout en s'accompagnant d'une mortalité deux fois moindre.

Des résultats non moins convaincants furent également obtenus avec l'Arc-en-Ciel et la Tète d'acier (S. Gairdneri).

Le " foie de mouton ", qui donna ensuite les meilleurs résultats, fut expérimenté cru ou cuit. Les résultats furent variables, souvent mème contradictoires et ceci, probablement, à cause de facteurs encore mal déterminés.

In essai de 103 jours avee des Truites de rivière montra l'avantage du foie de mouton cru sur le foie de mouton cuit ; la mortalité dans le premier lot, nourri au foie cru, étant quatre fois moindre que dans le lot nourri au foie cuit. Il en fut de même avec la Truite arc-en-ciel ; après 94 jours, la mortalité était moindre et la croissance plus forte dans le lot nourri au foie cru.

Il n'en est pas moins vrai que de nombreux pisciculteurs des EtatsInis emploient toujours avec succès le foie de mouton cuit comme aliment. Ces dernier résultats demandent donc confirmation.

Ine autre série d'essais eut pour objet de rechercher des aliments autres que les trois précédemment indiqués et susceptibles de donner, soit employés seuls, soit mélangés avec le régime normal, des résultats appréciables dans l'alimentation des alevins ou des adultes.

(1) Lumer Higgins. Progress in Biological Inquiries. Appendix VII to the Report of the United States Commissioner of Fisheries for 1927. - B. F. Doc. 1929.

(3) Bulletin Français de Pisciculture, $\mathrm{n}^{\circ}$ 6, Décembre 1928 . 
Trois substances donnèrent des résultats encourageants, parfois même supérieurs au régime carné. Ce sont : les déchets de Soja, les Crevettes séchées et les Moules d'eau douce.

Le Soja est une plante de la même famille que notre Haricot et cultivée dans toute l'Asie. Ses graines peuvent ètre consommées à l'état frais ou servent pour l'extraction de l'huile. Les résidus de cette opération sont, alors, employés comme tourteaux.

Ce sont ces tourteaux qui furent essayés. L'avantage de cet aliment était d'être très riche en protéine ressemblant davantage, par sa constitution, à une protéine animale qu'à une protéine végétale et, par conséquent, plus facilement assimilable.

Employé seul, le Soja ne se montra pas très favorable, mais, mélangé à de la viande dans les proportions de $\bar{c}$ c \% de Soja pour jo \% de viande et essayé sur alevins de Truites de lac et de Truites de ruisseau, il donna des résultats meilleurs, les lots en essai avant pu ôtre conservés sans pertes, bien que leur croissance fût, toutefois, légèrement inférieure à . celle des lots témoins.

Il est supposé que le Soja conviendrait mieux dans le régime alimenzaire des adultes et des Truites-portion ; des essais sont en cours.

Les déchets provenant des établissements de séchage de Crevettes ne donnèrent aucun résultat daus l'alimentation des jeunes alevins, probablement à cause de leur fort pourcentage en chitine inassimilable. Mais, à la Station de Holden, des Crevettes décortiquées et moulues après détrempage dans l'eau, furent administrées à de jeunes 'Iruites de 5 à 8 centimètres et plus. Employé seul, cet aliment fut défectueux et le fait se traduisit par une importante augmentation de la mortalité ; mais, mélangé avec $50 \%$ de foie de mouton, il se montra excellent en provoquant une croissance exceptionnellement rapide sans mortalité appréciable.

Enfin, la chair de Moule d'eau douce fut expérimentée, mais seulement sur une petite échelle ; les résultats furent particulièrement encourageants. Un lot d'alevins de "Tète d'acier ", nourri pendant 97 jours avec un régime constitué de $75 \%$ de cosur de boruf et de $25 \%$ de chair de Moule desséchée, présenta une mortalité deux fois moindre et une croissance d'un quart supérieure au lot témoin nourri uniquement de foie de bœuf.

De ces essais expérimentaux, poursuivis en Amérique, il convient surtout de retenir le principe et la méthode. Les pisciculteurs américains ont l'énorme avantage, grâce au développement intense de l'industrie des conserves de viande au Etats-L'nis, de pouvoir se procurer, aisément et à très bas prix, des abats dé choix. Il n'en est pas de même chez nous et les foies de bœuf ou de mouton, le cosur de bouf atteignent encore des prix tels que leur emploi reste pratiquement impossible pour le pisciculteur français. 
Celui-ci doit rechercher autre chose et, de plus en plus, il se reporte sur les déchets de Poisson : déchets bruts ou farines qui, jusqu'ici, ont paru donner des résultats appréciables.

Mais est-ce bien là le régirne rationnel, celui capable de donner le maximum de rendement avec le minimum de pertes et de frais? L'élevage expérimental pourrait seul nous le dire. In l'absence de toute Station de recherches officielles, il revient donc au pisciculteur de faire ces essais lui-même, à ses risques ei périls. Cés risques sont-ils si grands que cela et d'une expérience bien menée ne peut-il ensuite en tirer d'énormes avantages?

La méthode exige surtout une certaine patience, une grande attention et de la place vacante, car, tout bon pisciculteur connaît l'origine de ses alevins. Il lui est donc possible de répartir une certaine quantité d'alevins de mème origine, connue, en différents lots placés séparément, en les maintenant, toutefois, dans les mèmes conditions d'élevage, et, de les soumetlre à différents régimes alimentaires parfaitement définis. In lot témoin, soumis au régime alimentaire normal, permet de constater, au bout d'un temps plus ou moins longr : trois à six mois, quel est l'avantage de tel ou tel récrime en essai, quant à la mortalité, facilement contrôlable, et à la croissance, vérifiable aisément au cours ou en fin d'expérience.

Nous soumettons simplement cette suggestion aux Salmoniculteurs français.

\section{L'INFLUENGE DE LA LUMIÈRE SUR LES ALEVINS DE SALMONIDES}

Par M. JEAN LE GALL

Il est de connaissance courante, cher tous les pisciculteurs de métier que les bacs d'alevinage doivent itre protégés contre la lumière. lls sont, pour cela, généralement recouverts par des trappes mobiles.

Il n'en était pas moins intéressant de rechercher, scientifiquement, quelle pourait ètre l'influence de la lumière ou, plus exactement, l'influence des rayons ultra-violets sur la morlalité et la croissance des jeunes alevins.

Des essais furent tentés, dans ce sens aux Etats-L'nis, à la Station de Holden, sur des alevins de Truite de lac et de Truite arc-en-ciel.

Dans les différents compartiments d'un mème bassin, abondamment irrigué, furent placés différents lots de poissons de même origine : les uns dans des compartiments complètement recouverts de façon à empêcher la pénétration directe de la lumière, les autres dans des com- 